\title{
OPTIMAL TAXES AND THE STRUCTURE OF PREFERENCES
}

\begin{abstract}
By Angus Deaton ${ }^{1}$
If optimal tax theory is to be the basis for calculating tax rates, a close understanding is required of the relationship between the structure of preferences and the configuration of optimal tax rates. Otherwise hypotheses chosen by the econometrician for practical convenience may completely determine the results, independently of measurement. This paper explores the relationship between various types of separability, particularly weak and implicit separability, and optimal tax rates in the various models discussed in the literature. The use of distance functions and the Antonelli matrix provides a significant unification of previously disparate results.
\end{abstract}

IN THE FINAL ANALYSIS, optimal tax theory should be the basis for actual calculation of tax rates. Although recently there have been great advances in theoretical results and in our understanding of their meaning, we are still some way from a working knowledge of whether uniform commodity taxes are in practice optimal or, if not, which commodities should be discriminated against. Present theoretical formulae do not yield clear-cut results except in special cases and it has recently become clear that optimal rates depend crucially on the detailed structure of consumer preferences. For example, Atkinson and Stiglitz [3]' show that with an optimal nonlinear income tax, discriminatory commodity taxes are only necessary to the extent that individual commodities are not weakly separable from leisure. More recently, Deaton [6] has shown that a similar result holds for what is perhaps the most interesting of the standard models, that where there are many consumers and only a linear income tax and proportional commodity taxes are allowed. In this case, separability between goods and leisure, together with linear Engel curves for goods, removes the need for differential commodity taxation. In consequence, nothing can be learned about commodity taxes from consumer demand studies in which commodity demands are explained conditionally on total expenditure and commodity prices and which assume linear Engel curves. All such studies require separability from leisure as a maintained hypothesis and so are consistent with uniform commodity taxation. These results suggest that the prospects for meaningful empirical calculations of tax rates are bleak. Econometricians estimating commodity demand and labor supply equations make generous use of separability assumptions to enable estimation at all. In consequence, it is likely that empirically calculated tax rates, based on econometric estimates of parameters, will be determined in structure, not by the measurements actually made, but by arbitrary, untested (and even unconscious) hypotheses chosen by the econometrician for practical convenience.

To remedy this situation, and as a prelude to fruitful empirical work, it is necessary to have a more explicit understanding of how preference structure affects optimal tax rates. Such is the object of this paper. Three different

\footnotetext{
${ }^{1}$ The ideas in this paper were first presented to the Warwick Summer Workshop on public economics, July, 1978. I should like to thank Jim Mirrlees for his detailed comments on an earlier version; Avinash Dixit, Terence Gorman, Peter Hammond, Frank Hahn, and Tony Jackson also made helpful suggestions. Remaining errors are my own.
} 
frameworks are considered: the one consumer Ramsey-rule economy, the many consumer economy with linear income tax and proportional commodity taxes, and the continuum of consumers economy with general nonlinear taxes. In each framework separability assumptions will be varied parametrically and the effects on the structure of taxes deduced. Several of the results in the paper are well known. However, by adopting a rather different approach from normal, a unity in previously rather disparate results is revealed, and this greatly helps to simplify the analysis.

The first section of the paper is devoted to prior methodological issues. Much use is made in the main analysis of inverse compensated demand functions relating prices to utility and to quantities consumed. Although such functions were discussed by Hicks [15], they are relatively unfamiliar and a brief discussion of their properties is given. In the present context, their usefulness lies in the ease with which they enable the change in the marginal rate of substitution between two goods to be broken up into movements along an indifference curve and movements outwards or inwards along a quantity ray. This decomposition is crucial since it turns out that, in all the models considered, differences in tax rates between commodities can be related to the effects of leisure on the marginal rate of substitution between the corresponding commodities. These effects are crucially dependent on separability assumptions between goods and leisure, and the consequences of two types of separability - weak separability and implicit or quasi-separability - are extensively explored. Section 2 derives the optimal tax formulae for each framework in a common format and the implications of separability are discussed. In the case which is analytically the most difficult, the model with many consumers, a linear income tax and proportional commodity taxes, approximation formulae are used. These replace the demand functions by local linearizations which have the effect of allowing perfect aggregation over households. Section 3 is a preliminary discussion of empirical implementation of the results. Section 4 summarizes the main findings.

\section{COMPENSATED INVERSE DEMANDS AND THE ANALYSIS OF SEPARABILITY}

With any indifference curve $u$ and quantity ray $\vec{q}$ (the arrow indicates that scale is unimportant) it is possible, given convex preferences, to associate a vector of price to expenditure ratios $x^{-1} p$ such that a consumer on $u$ faced with prices proportional to $x^{-1} p$ will purchase a quantity bundle along $\vec{q}$. Figure 1 illustrates. We write these functions

$$
x^{-1} p_{i}=a_{i}(u, q)
$$

where $a_{i}$ is homogeneous of degree zero in $q$. These functions are dual to the more familiar Hicksian compensated demands

$$
q_{i}=h_{i}(u, p) \text {. }
$$

As is well known, the Hicksian demands are the partial derivatives with respect 


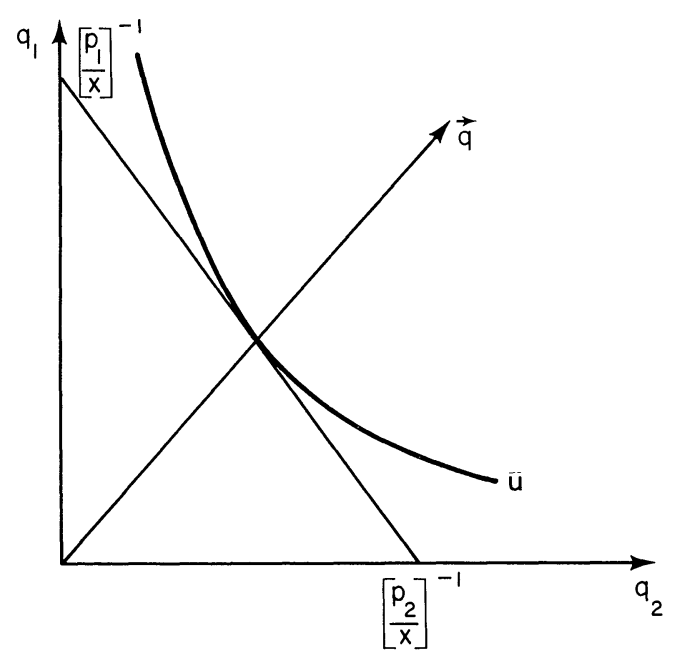

FIGURE 1.-Compensated inverse demands.

to $p$ of the cost function, $c(u, p)$, defined by

$$
c(u, p)=\min _{q}\{p \cdot q ; \quad v(q) \geqslant u\}
$$

for direct utility functions $v(q)$. Similarly, the $a_{i}(u, q)$ functions are the partial derivatives with respect to $q$ of the distance function, $d(u, q)$, defined by

$$
d(u, q)=\max \{\lambda ; v(q / \lambda) \geqslant u\} .
$$

The distance function $d(u, q)$ is continuous in its arguments, is decreasing in $u$, and increasing, linearly homogeneous, and (if $v(q)$ is quasi-concave) concave, first and second differentiable almost everywhere in $q$. The functions $a_{i}(u, q)$ thus possess derivatives almost everywhere and we write

$$
a_{i j}(u, q)=\frac{\partial a_{i}(u, q)}{\partial q_{j}}=\frac{\partial^{2} d(u, q)}{\partial q_{i} \partial q_{j}} .
$$

The elements $a_{i j}$ form the Antonelli matrix which is clearly symmetric and negative semi-definite. (For a full analysis of these functions and their properties, see Deaton [5].)

The Antonelli matrix is useful in tax theory since it can be used to "invert" its generalized inverse, the Slutsky matrix, and thus to rewrite any expressions involving substitution effects. The inversion formula used in the next section is derived from the identity

$$
p_{i} / c(u, p)=a_{i}\{u, \nabla c(u, p)\},
$$

where $\nabla c(u, p)$ is the gradient of $c$, i.e. the Hicksian demands $h(u, p)$. Multiplying through by $c(u, p)$ and differentiating with respect to $p_{j}$, application of the 
chain rule gives, for all $i, j$,

$$
\delta_{i j}=c(u, p) \sum_{k} a_{i k} s_{k j}+c_{j}(u, p) a_{i}\{u, \nabla c(u, p)\}
$$

for substitution responses $s_{k j}$ and Kronecker delta $\delta_{i j}$, so that, since $c(u, p)=x$, $c_{j}(u, p)=q_{j}$ and $a_{i}\{u, \nabla c(u, p)\}=p_{i} / x$, we may write

$$
x \sum_{k} a_{i k} s_{k j}=\delta_{i j}-p_{i} q_{j} x^{-1} .
$$

For later reference, this may be written in obvious matrix notation as

$$
x A S=I-p q^{\prime} x^{-1}
$$

where $I$ is the identity matrix, $p, q$ are column vectors, and a prime denotes transposition. Note that $\left(7^{\prime}\right)$, together with the homogeneity requirement $A q=0$, defines $A$ given $S, p, q$, and $x$. Explicit formulae for calculating $A$ will be given later; for the moment, (7) is all that is required for the theory.

It will be convenient to treat leisure asymmetrically and we decompose the $q$ vector into $\left(q_{0}, q\right)$ with leisure denoted as good 0 ; the corresponding price is $p_{0}$. Each consumer faces a budget constraint

$$
p_{0} q_{0}+\sum p_{k} q_{k}=p_{0} T+b \equiv x
$$

for time endowment $T$ and transfer income $b$. The quantity $x$ is "full" income or total expenditure. With these interpretations of $q, p$, and $x$, the foregoing analysis goes through with all indices running from 0 to $n$.

If we confine the analysis to situations where all commodities are purchased, we can write the marginal rate of substitution between goods $i$ and $j, \mathrm{MRS}_{i j}$, in each of the following ways:

$$
\operatorname{MRS}_{i j}=p_{i} / p_{j}=u_{i} / u_{j}=a_{i}(u, q) / a_{j}(u, q),
$$

for marginal utilities $u_{i}=\partial v / \partial q_{i}$. By using $a_{i} / a_{j}$ rather than $u_{i} / u_{j}$ for $\mathrm{MRS}_{i j}$, we can decompose changes in $\mathrm{MRS}_{i j}$ into those along an indifference curve and those out along a ray. This is precisely analogous to the usual decomposition into income and substitution effects in the dual space. In this latter, compensation is ensured by a change in income or equivalently, a proportional change in prices, while in the primal space, compensation is by a proportional change in quantities. In particular, we shall be interested in the effects of changes in leisure and we write

$$
\frac{\partial \log \left(u_{i} / u_{j}\right)}{\partial q_{0}}=\frac{\partial \log \left(a_{i} / a_{j}\right)}{\partial q_{0}}+\frac{\partial \log \left(a_{i} / a_{j}\right)}{\partial u} \cdot \frac{\partial u}{\partial q_{0}} .
$$

Hicks [15] defines goods as q-complements if $a_{i j}>0$ (the marginal valuation of $i$ rises along an indifference curve as more $j$ is consumed) and as $q$-substitutes if $a_{i j}<0$. Hence we can say that $\partial \log \left(a_{i} / a_{j}\right) / \partial q_{0}$ is positive or negative as $i$ is more 
complementary or substitutable with leisure in the Hicks " $q$ " sense. Except as indicated, all further reference to complements and substitutes in this paper will be in this sense; the reader should be warned that, in general, these definitions do not correspond to the usual Hicks-Allen or " $p$ " definition through the Hessian of the cost function. The last term in (9) can also be given a sensible, although nonstandard, interpretation. If $\partial \log \left(a_{i} / a_{j}\right) / \partial u$ is positive, the marginal valuation of $i$ rises relative to $j$ as the consumer becomes better off; in this case it seems reasonable to say that $i$ is relatively luxurious compared with $j$. Once again, this definition of relative luxury is adopted for the rest of the analysis.

Equation (9) takes a simpler form under the two types of separability we shall consider. Goods $i$ and $j$ are said to be weakly separable from leisure if $\mathrm{MRS}_{i j}$ is independent of leisure, $q_{0}$. If this is true for all $i$ and $j$, utility must take the form

$$
v\left(q_{0}, q\right)=v^{*}\left(q_{0}, \phi(q)\right)
$$

for some function $\phi()$ which is, in turn, equivalent to the proposition that for $i=1, \ldots, n, q_{i}$ can be written as a function of $p \cdot q$ and $p$ alone, so that changes in $q_{0}$ exert only income effects on the choice of commodities. Under weak separability, the left-hand side of (9) is zero so that, for $i, j=1, \ldots, n$,

$$
\frac{\partial \log a_{i} / a_{j}}{\partial q_{0}} \propto-\frac{\partial \log a_{i} / a_{j}}{\partial u}
$$

assuming $\partial u / \partial q_{0}>0$. Hence, if $i$ and $j$ are weakly separable from leisure, the commodity which is relatively luxurious must also be the more substitutable with leisure.

In contrast to the above, goods $i$ and $j$ are said to be implicitly or quasiseparable (see Gorman $[12,13]$ ) from leisure if $\mathrm{MRS}_{i j}$ is independent of $q_{0}$ along an indifference curve, if the consumer is compensated for the change in $q_{0}$ by a proportional change in the vector $\left(q_{0}, q\right)$. If this is true for all $i$ and $j$, the distance and cost functions must take the forms

$$
\begin{aligned}
& d\left(u, q_{0}, q\right)=d^{*}\left(u, q_{0}, \theta(u, q)\right), \\
& c\left(u, p_{0}, p\right)=c^{*}\left(u, p_{0}, \eta(u, p)\right),
\end{aligned}
$$

for linearly homogeneous concave functions $\theta()$ and $\eta()$. For proofs of the equivalence of (11) and (12), see Gorman [13], McFadden [16] or Deaton and Muellbauer [8, pp. 134-5]. In this case, (11) or (12) are equivalent to the proposition that for $i=1, \ldots, n, p_{i} q_{i} / p \cdot q$, the commodity budget shares, can be written as a function of $u$ and $p$ alone. Hence, compensated changes in $p_{0}$ affect commodity demands proportionately. Under quasi-separability, the first term on the right-hand side of (9) is zero so that, for $i, j=1, \ldots, n$,

$$
\frac{\partial \log \left(u_{i} / u_{j}\right)}{\partial q_{0}} \propto \frac{\partial \log a_{i} / a_{j}}{\partial u} .
$$

There is no obvious presupposition in favor of one or the other of these two 
types of separability given intuitive notions of what separability between goods and leisure might mean. It is certainly not true that either one is more restrictive than the other in terms of limiting the number of possible behavioral responses. Nevertheless, they are quite different. If leisure is both weakly and quasiseparable from goods, we have immediately from (9) that $\partial \log \left(a_{i} / a_{j}\right) / \partial u=0$. Since $\partial \log \left(a_{i} / a_{j}\right) / \partial q_{0}=0$, there exist functions $\mu\left(u, q_{0}\right)$ and $b_{i}(q)$, the latter homogeneous of degree zero such that $a_{i}\left(u, q_{0}, q\right)=\mu\left(u, q_{0}\right) b_{i}(q)$. Hence, integrating, $d\left(u, q_{0}, q\right)=\mu\left(u, q_{0}\right) b(q)$ where $b(q)$ is a linearly homogeneous function satisfying $b_{i}(q)=\partial b / \partial q_{i}$. Since, from (4), $d\left(u, q_{0}, q\right)=1$ defines $v(q)$, we have $v(q)=f\left(q_{0}, b(q)\right)$ so that utility is weakly homogeneously separable. Hence, the intersection of weak and implicit separability is the homogeneous case which implies that the total expenditure elasticities of all goods are unity.

\section{OPTIMAL TAX FORMULAE}

In this section, we make no attempt to go beyond the standard first-order conditions which are familiar in the literature. As has been emphasized by Mirrlees [17] these conditions are not only not sufficient for a tax optimum, they may not even be necessary. The implications deduced are thus properties of the conventional formulae and not necessarily of the tax optimum. Nevertheless, one might hope that some tax optima, at least, satisfy these conditions.

\section{Case 1: The One Consumer Economy}

This is an highly artificial model but one which serves as a benchmark for other results. One single household, preferences represented by the cost function $c\left(u, p_{0}, p\right)$, has full income $p_{0} T$ since no lump-sum transfers are allowed. The government wishes to raise a fraction $\rho$ of full income by means of commodity taxes alone, the zero tax on leisure being an arbitrary normalization. The problem is thus to maximize $u$ subject to (a) $c\left(u, p_{0}, p\right)=p_{0} T$ and (b) $\sum t_{k} h_{k}(u$, $\left.p_{0}, p\right)=\rho p_{0} T$. In this and subsequent analysis, it is assumed either that constant returns prevail or that all profits are taxed at 100 per cent. Consequently, $p$ varies one for one with the taxes $t$. The maximization problem has the familiar solution

$$
\sum_{k=1}^{n} s_{i k} t_{k}=\alpha q_{i} \quad(i=1, \ldots, n),
$$

for some magnitude $\alpha$ not indexed on $i$. Since $t_{0}$ is zero, the summation can be extended from $k=1, \ldots, n$ to $k=0, \ldots, n$ so that (14) can be rewritten, for some $\beta$,

$$
\sum_{0}^{n} s_{i k} t_{k}=\alpha q_{i}+\beta \delta_{i 0}
$$

$$
(i=0, \ldots, n)
$$

Multiply (15) by $a_{j i}$, sum over $i$, and apply the inversion formula (7). Hence

$$
x \sum_{i} \sum_{k} a_{j i} s_{i k} t_{k}=\sum_{k}\left(\delta_{j k}-x^{-1} p_{j} q_{k}\right) t_{k}=\sum_{i} x a_{j i}\left(\alpha q_{i}+\beta \delta_{i 0}\right) .
$$


But by the homogeneity of the $a_{i}$ functions $\sum_{i} a_{j i} q_{i}=0$, and using $t_{0}=0$, $\beta=-a_{0} \rho / a_{00}$. Making the substitutions

$$
\frac{t_{j}}{p_{j}}=\rho\left\{1-\frac{a_{j 0} a_{0}}{a_{j} a_{00}}\right\}
$$

or alternatively

$$
\frac{t_{i}}{p_{i}}-\frac{t_{j}}{p_{j}}=\left(\frac{-\rho a_{0}}{a_{00}}\right) \frac{\partial \log a_{i} / a_{j}}{\partial q_{0}} .
$$

(Equations (17) and (18) may be derived more elegantly by specifying the tax problem directly in terms of the distance function-see Deaton [5, p. 403]rather than by working from (14). I have pursued the less direct procedure here to illustrate the general applicability of the inversion technique to formulae involving the substitution matrix.)

Note that the quantity $\left(-\rho a_{0} / a_{00}\right)$ is positive if $\rho$ is positive since $d\left(u, q_{0}, q\right)$ is concave. Hence, the Ramsey rule in form (18) says simply that goods which, at the tax optimum, are relatively complementary with leisure bear the higher tax. No other considerations are directly relevant. The implications of separability for the Ramsey rule are thus quite clear. Under quasi-separability the right-hand side of (18) is zero so that no discriminatory taxes are required, instead a uniform tax on commodities or, its equivalent, a proportional income tax should be imposed. Under weak separability, however, by (11), the Ramsey rule implies that those goods which are relative necessities at the optimum should be taxed relatively highly. We shall describe this case by saying that commodity taxes are "regressive." Hence, if the Ramsey rule is used to calculate commodity taxes from a model such as the linear expenditure system which is strongly, and hence, $a$ fortiori, weakly separable, regressive commodity taxes must always result; see e.g. the calculations in Atkinson and Stiglitz [2]. However, there is no general presupposition that the Ramsey rule is equity disregarding in this way. Indeed, it is not implausible to suppose that, in general, relative luxuries are also relatively complementary with leisure. In this case, (18) would produce a progressive commodity tax structure.

\section{Case 2: An Unidimensional Continuum of Consumers with Nonlinear Taxes-Atkinson and Stiglitz [3]}

It is here assumed that nonlinear taxes can be levied on all goods although, once again, leisure is by convention untaxed. Consumers thus face prices $z_{i}+\tau_{i}\left(q_{i}\right)$ for producer prices $z_{i}$ and tax functions $\tau_{i}\left(q_{i}\right)$ dependent on $q_{i}$ alone. Consumers are indexed by the variable $p_{0}$ which has density function $f\left(p_{0}\right)$ over consumers. For convenience, the social welfare function is assumed to be additive over consumers so that the problem is to maximize

$$
W=\int w(u) f\left(p_{0}\right) d p_{0}
$$


subject to the household budget constraint

$$
\sum\left\{z_{i}+\tau_{i}\left(q_{i}\right)\right\} q_{i}+p_{0} q_{0}=p_{0} T,
$$

the government revenue constraint

$$
\int \sum \tau_{i}\left(q_{i}\right) q_{i} f\left(p_{0}\right) d p_{0}=R
$$

the utility constraint

$$
d\left(u, q_{0}, q\right)=1 \equiv q_{0}=\xi(u, q),
$$

where the alternative form is obtained by inversion, and finally the derivative constraint

$$
\frac{\partial u}{\partial p_{0}}=\frac{\left(T-q_{0}\right)}{h_{u}}=\frac{-a_{0}\left(T-q_{0}\right)}{a_{u} p_{0}}
$$

where $h_{u}=\partial c / \partial u$ and $a_{u}=\partial d / \partial u$. Equation (23) derives from differentiation of $c\left(u, p_{0}, p\right)=p_{0} T$ with respect to $p_{0}$, differentiation of $d\left(u, q_{0}, q\right)=1$ with respect to $q_{0}$, and finally use of the first-order condition $\partial v / \partial q_{0}=\lambda p_{0}=p_{0} / h_{u}$. If (22) is used to substitute for $q_{0}$, the problem (19) to (23) is susceptible to straightforward application of the maximum principle if $u$ and $q$ are chosen as instruments. Routine manipulation then yields, in accordance with the Atkinson and Stiglitz result, writing $t_{i}=\tau_{i}^{\prime}\left(q_{i}\right)$ and $p_{i}=z_{i}+t_{i}$,

$$
\frac{t_{i}}{p_{i}}-\frac{t_{j}}{p_{j}} \propto \frac{\partial \log u_{i} / u_{j}}{\partial q_{0}},
$$

the only difference from (18) being that the leisure derivative of $\mathrm{MRS}_{i j}$ is no longer compensated. As has been much commented upon, (24) implies that, under weak separability, no discrimination is required so that, in the presence of an optimal nonlinear income tax, commodity taxation is unnecessary. However, if instead leisure is implicitly separable from goods, (24) together with (13) imply that progressive (nonlinear) commodity taxation is desirable. Since this is not likely to be generally feasible, it may well be desirable to have, for example, uniform quotas of certain goods; see Roberts [20], Guesnerie [14]. Once again, the tax prescription is very sensitive to what might reasonably be thought of as small changes in the precise definition of separability.

\section{Case 3: Many (Discrete) Consumers, Linear Income Tax and Proportional Commodity Taxes}

This third model, unlike the Ramsey model, explicitly takes equity into account, but unlike the previous case allows only taxes that it is clearly feasible to collect. Once again, leisure is untaxed although in this case the government can pay (levy) a lump-sum benefit (tax) on all consumers which must be the same for 
all. Denote this amount by $b$. The maximization problem is then to maximize

$$
W=\Omega\left(u^{1}, u^{2}, \ldots, u^{N}\right)
$$

subject to the utility constraint

$$
p_{0}^{h} T+b=c^{h}\left(u^{h}, p_{0}^{h}, p\right)
$$

and the revenue constraint

$$
\sum_{h} t \cdot q^{h}=N b+R
$$

for $N$ consumers with government revenue $R$ required for purposes other than the benefits $b$. The instruments for this problem are the $N u^{h}$ 's, the tax rates $t$ (or prices $p$ ), and the benefit level $b$. Write the Lagrangian

$$
L=W+\sum \lambda^{h}\left\{p_{0}^{h} T+b-c^{h}\left(u^{h}, p_{0}^{h}, p\right)\right\}+\xi\left\{\sum_{h} t \cdot q^{h}-N b-R\right\} .
$$

Differentiating with respect to $b$ gives immediately $\xi=\bar{\lambda}$, the mean of the $\lambda^{h}$ 's, so that, differentiating with respect to $u^{h}$ and rearranging

$$
\lambda^{h}=\frac{\partial \Omega}{\partial b^{h}}+\bar{\lambda} \frac{\partial R^{h}}{\partial b}
$$

where $\partial \Omega / \partial b^{h}=\partial \Omega / \partial u^{h} \cdot \partial u^{h} / \partial b$ is the (gross) marginal social utility of income to individual $h$, and $R^{h}=t \cdot q^{h}$, the taxes paid by $h$. Since $\bar{\lambda}=\xi$ is the marginal social utility of government revenue, $\lambda^{h}$ is interpretable-see Diamond [10]-as the net marginal social utility of income paid to $h$. Differentiation of (28) with respect to $t_{i}$ gives

$$
\frac{1}{N} \sum_{h} \sum_{k} s_{i k}^{h} t_{k}=\sum_{h} \theta^{h} q_{i}^{h}-\frac{1}{N} \sum q_{i}^{h}
$$

where $\theta^{h}=\lambda^{h} / N \bar{\lambda}$ is $h$ 's net marginal social utility share. (N.B. $\sum \theta^{h}=1$.) Equation (30) is equivalent to the covariance formula discussed by Diamond [10] and later writers. For the present purposes, rewrite the equation as, for $i=$ $1, \ldots, n$,

$$
\sum_{k=1}^{n} \bar{s}_{i k} t_{k}=q_{i}^{*}-\bar{q}_{i}
$$

where $\bar{s}_{i j}$ and $\bar{q}_{i}$ are the means of $s_{i j}^{h}$ and $q_{i}^{h}$, and $q_{i}^{*}$ is the $\theta^{h}$-weighted average of $q_{i}^{h}$. Note that if $\theta^{h}$ contains some egalitarian bias, $q_{i}^{*}$ will have a greater bias towards the consumption of the poor so that (31) will imply that taxes are such that a uniform intensification in taxes should bear more heavily on the consumption of luxuries. What this implies for taxes, however, depends crucially on the precise configuration of preferences.

One particularly simple solution occurs if we make the frequently met assump- 
tion that cross-substitution effects between goods are zero so that all commodities are $(p-)$ substitutes for leisure and the cost functions take the form $\sum_{k} \gamma_{k}^{h}\left(u^{h}, p_{0}^{h}, p_{k}\right)$ for $n$ functions $\gamma_{k}^{h}()$. In this case, the average $\bar{s}_{i k}$ will also be zero so that (31) simplifies to

$$
\frac{t_{i}}{p_{i}}=\frac{\left(1-\left(q_{i}^{*} / \bar{q}_{i}\right)\right)}{\left(-\bar{e}_{i i}\right)}
$$

where $\bar{e}_{i i}$ is the average compensated own-price elasticity for good $i$. The numerator of $\left(31^{\prime}\right)$ is a "social" luxury index for good $i$ so that the formulae represents a ratio of equity to efficiency effects evaluated at the tax optimum.

A more general solution cannot be obtained by applying the inversion formulae to (31) directly since, without restrictions on preferences, it is not always possible to find preferences which for $\bar{p}_{0}$ (or any other level of $p_{0}$ ) will generate $\bar{s}_{i j}$ and $\bar{q}$. However it is possible to justify such aggregation as an approximation. In particular, if (a) commodity demands are a linear function of both the wage rate $p_{0}$ and full income $\left(p_{0} T+b\right)$, and (b) the value of labor supply, $p_{0}\left(T-q_{0}\right)$, is also a linear function of the same variables, then perfect aggregation with $\bar{p}_{0}$ will generate demands $\bar{q}$ and a value of labor supply equal to the mean of $p_{0}\left(T-q_{0}\right)$. Further, if $p_{0}^{*}$ is defined with the same weights as $q^{*}$, i.e. $p_{0}^{*}=\sum \theta^{h} p_{0}^{h}$, then a consumer with $p_{0}^{*}$ will generate demands $q^{*}$. The preferences which permit this are given by Muellbauer [18] and are defined by the cost function

$$
c\left(u, p_{0}, p\right)=u a(p) p_{0}^{\delta}+b(p) p_{0}+d(p)
$$

where $a(p), b(p)$, and $d(p)$ are functions homogeneous of degrees $(1-\delta), 0$, and 1 respectively, and $\delta$ is a parameter, $0<\delta<1$. It can easily be checked that (32), like the Gorman polar form of which it is an extension (see Diewert [11]), is a flexible functional form in that it can offer a first-order approximation at $\bar{p}_{0}$ to whatever are the true average demands. In fact, I shall need the somewhat stronger assumption that the linear demands from (32) adequately approximate the true demands for values of $p_{0}$ from $p_{0}^{*}$ to $\bar{p}_{0}$. Clearly this will hold either if (32) is exact or if $\bar{p}_{0}$ and $p_{0}^{*}$ are sufficiently close to one another. Further formulae in this section are derived on the assumption that this approximation holds good.

The linearization embodied in (32) implies that $\bar{s}_{i j}$ is the substitution term and $\bar{q}_{i}$ the consumption of an individual with wage $\bar{p}_{0}$, while $q_{i}^{*}$ is the consumption of an individual with wage $p_{0}^{*}=\sum \theta^{h} p_{0}^{h}$. Hence, the effect of the perfect aggregation assumption is to reduce the number of consumers from $N$ to two, one with mean wage $\bar{p}_{0}$ and the other with the "socially representative wage" $p_{0}^{*}$. Where $p_{0}^{*}$ lies in the wage distribution depends on the social welfare function (and the shape of Engel curves). If social preferences are such that the marginal social utility of money $\left(\partial \Omega / \partial b^{h}\right)$ is constant, and if all households have parallel linear Engel curves-assumed by (32) in any case- $\left(\partial R^{h} / \partial b=\right.$ const.), then $\bar{p}_{0}=p_{0}^{*}$. Otherwise, some egalitarian bias will make $p_{0}^{*}>\bar{p}_{0}$ with $p_{0}^{*}$ tending to the minimum wage as the social welfare function tends to maximin. 
Armed with these assumptions, the Antonelli matrix corresponding to the average consumer can be used to invert (31). Dropping the bars, since everything written without an asterisk is now a mean, (31) can be rewritten, for $i=0$, $1, \ldots, n$,

$$
\sum_{k=0}^{n} s_{i k} t_{k}=q_{i}^{*}-q_{i}+\delta_{i 0} \beta
$$

so that we can invert using $a_{j i}$ as before and solve out for $\beta$ using $t_{0}=0$. Writing $x=p_{0} T+b$ and $\rho=t \cdot q / x$, we have

$$
\frac{t_{i}}{p_{i}}-\frac{t_{j}}{p_{j}}=\beta \frac{\partial \log a_{i} / a_{j}}{\partial q_{0}}+\sum_{k} \frac{\partial \log a_{i} / a_{j}}{\partial q_{k}}\left(q_{k}^{*}-q_{k}\right)
$$

where

$$
\beta=\frac{-a_{0}}{a_{00}}\left\{\rho-\sum_{k} \frac{a_{0 k}}{a_{0}}\left(q_{k}-q_{k}^{*}\right)\right\} .
$$

Note that (34) and (35) are general results which can be interpreted even if the approximation (32) does not hold. For if representative preferences do not exist, (34) and (35) could be derived using the generalized inverse of the average Slutsky matrix derived directly from equation (7); see also equation (43) below. However, such a generalized inverse would not be the Antonelli matrix corresponding to any utility function, nor would there exist the corresponding compensated inverse demand functions. With the approximation (32), direct use can be made of the $a_{i}(u, q)$ functions for mean $u$ and $q$. Further, since the approximation is likely to be more accurate the closer is $p_{0}$ to $p_{0}^{*}$, it is natural to linearize the inverse demands between $q$ and $q^{*}$. As we shall see, this leads to simpler forms for (34) and (35). In particular, since $a_{i} / a_{j}=p_{i} / p_{j}$ which, for $i, j \neq 0$ is the same for all consumers, $d\left(\log a_{i} / a_{j}\right)=0$ so that

$$
\sum_{k=0}^{n} \frac{\partial \log a_{i} / a_{j}}{\partial q_{k}} d q_{k}+\frac{\partial \log a_{i} / a_{j}}{\partial u} d u=0 .
$$

Hence, using the assumed closeness of $q$ and $q^{*}$, (34) becomes

$$
\frac{t_{i}}{p_{i}}-\frac{t_{j}}{p_{j}}=\beta \frac{\partial \log a_{i} / a_{j}}{\partial q_{0}}+\frac{\partial \log a_{i} / a_{j}}{\partial u}\left(u-u^{*}\right) .
$$

Similarly, $\beta$ can be simplified by using $a_{0}=p_{0} x^{-1}=p_{0} /\left(p_{0} T+b\right)$ so that $d a_{0}=b d p_{0} /\left(p_{0} T+b\right)^{2}$ and using $d a_{0}=\sum a_{0 k} d q_{k}+a_{0 u} d u$, we have

$$
\beta=\left(\frac{-a_{0}}{a_{00}}\right)\left\{\rho-\left[\frac{b}{p_{0} T+b}+\frac{a_{0 u}}{a_{u}}\left(T-q_{0}\right)\right]\left(\frac{p_{0}-p_{0}^{*}}{p_{0}}\right)\right\}
$$

where I have used $u-u^{*} \simeq-\left(T-q_{0}\right) a_{0}\left(p_{0}-p_{0}^{*}\right) / a_{u} p_{0}$ which could also be used to replace $\left(u-u^{*}\right)$ in (37) by $\left(p_{0}-p_{0}^{*}\right) / p_{0}$. Note that $\left(u-u^{*}\right)$ is a measure of post-tax inequality-since $u$ and $u^{*}$ are average and socially representative 
utility at the tax optimum-while $\left(p_{0}-p_{0}^{*}\right) / p_{0}$ is pre-tax inequality. From the formula linking them, it is clear that if one is positive, so is the other.

Each term in (38) taken separately is positive so that the sign of $\beta$ depends on the balance between $\rho$, government revenue as a proportion of potential GDP, and $\left(p_{0}-p_{0}^{*}\right) / p_{0}$, the measure of pre-tax social inequality, multiplied by the (positive) term in square brackets. Equations (37) can now be examined through a series of special cases.

Case 1-Quasi-separability: Only the second term in (37) is nonzero, so that provided there is some initial (perceived) inequality, the commodity tax system is progressive.

Case 2-Weak Separability: In this case (37) has a uniform tax solution with the value of the tax rate chosen to satisfy $u-u^{*}-\beta \partial u / \partial q_{0}=0$. This is the case examined in Deaton [6] and it should be emphasized that this uniformity result requires the linear Engel curve assumption which underlies the current analysis. Given the cost function (32), explicit solutions for the tax rate are possible provided we deal only with rank-order social welfare functions; the topic is too extensive to be discussed here and will be presented in a separate paper.

Case 3-No Inequality: There will be no post-tax inequality $\left(u=u^{*}\right)$ if and only if there is no pre-tax inequality $\left(p_{0}=p_{0}^{*}\right)$ at least as perceived by the social welfare function. If this is so (37) reduces to the Ramsey rule (18). Note the special place occupied by good 0 , leisure. This is not because of equity effects operating through differences in $p_{0}^{h}$ since, in this case, there are no such differences. Rather the asymmetry is due to the numeraire role of labor (or leisure). Since leisure is untaxed, government revenue is implicitly measured in labor units so that by taxing complements with the revenue good, taxation is rendered easier. In general, the government will presumably wish to purchase goods other than labor and this would lead to a different tax rule. For example, a king who must pay a tribute of oxen to a neighboring conqueror would do well to levy relatively high taxes on goods complementary with oxen.

Case 4 -Pure Redistribution: With $\rho=0, \beta$ is unambiguously negative and commodity taxes can either be progressive or regressive. The equity effect in favor of high taxation on luxuries operates as in Case 1 but there is also a presumption in favor of high taxation on goods which are complementary with work (substitutable for leisure). This is presumably because of the comparative advantage enjoyed by the rich in work, so that post-tax inequality is reduced by discouraging it. In any case goods which are both luxuries and complementary with work should be highly taxed (first-class travel, academic books?).

Case 5: In general all these forces operate simultaneously. Note, in particular that (38) suggests (but does not prove) that the tax structure may switch from progressivity to regressivity as the government revenue requirement increases.

Note finally the clear family resemblance between the optimal tax formulae in the three models, equations (18), (24), and (37). All three take the general form

$$
\frac{t_{i}}{p_{i}}-\frac{t_{j}}{p_{j}}=\theta_{1} \frac{\partial \log \left(a_{i} / a_{j}\right)}{\partial q_{0}}+\theta_{2} \frac{\partial \log \left(a_{i} / a_{j}\right)}{\partial u}
$$


for some choice of $\theta_{1}$ and $\theta_{2}$. (In (18), $\theta_{2}=0$; in (24) $\theta_{2}=\theta_{1}\left(\partial u / \partial q_{0}\right)$; in (37) $\theta_{1}=\beta, \theta_{2}=u-u^{*}$.) Alternatively, (9) can be used to rewrite (37) as the sum of the compensated and uncompensated derivatives of the marginal rate of substitution between $i$ and $j$ with respect to leisure. By contrast, equation (18), the Ramsey rule, depends only on the compensated derivative, while equation (24), the Atkinson-Stiglitz formula, depends only on the uncompensated derivative. This suggests a fundamental unity between the results which has been previously obscured by the fact that Cases 1 and 3 are usually dealt with in price space-giving a solution in quantity space while Case 2 is dealt with in quantity space with its solution in price space. In substantive terms, (39) suggests that differential commodity taxes are related quite generally both to the degree of luxury of the goods and to the degree of complementarity with leisure. The former is a pure equity effect while the latter has aspects of both equity and efficiency, one operating to tax complements with work, the other to tax complements with leisure.

\section{SOME EMPIRICAL CONSIDERATIONS}

If the tax formulae of the previous section are to be implemented we need empirical estimates of the Antonelli matrix and of the derivatives of the inverse compensated demands with respect to utility. Econometricians are much more used to measuring the dual concepts, the parameters of the Hicksian demands. In principle, it is possible to estimate inverse demand functions in which prices are regressed on quantities consumed. But, in most modern economies, and isolated commodities apart, it is not very sensible to regard quantities consumed as exogenous to consumers with price determined by income and tastes. Clearly then, we must estimate the commodity demands and then calculate from these the quantities we require.

Assume that we have estimated a system of demand equations, including the demand for leisure, so that estimates of the Slutsky matrix $S$ (as functions of $x$ and $p$ ) and of the income derivatives are available. Recalling $\left(7^{\prime}\right)$, i.e.

$$
x A S=I-p q^{\prime} x^{-1}
$$

we have also, from the homogeneity of $d(u, q)$ and $c(u, p)$

$$
A q=0, \quad S p=0 .
$$

Hence, $A\left\{x S+q q^{\prime}\right\}=x A S=\left(I-p q^{\prime} x^{-1}\right)$ so that

$$
A=\left(I-x^{-1} p q^{\prime}\right)\left(x S+q q^{\prime}\right)^{-1} .
$$

This formula is practical but not very elegant since it disguises the symmetry of the relationship. This can be re-established by using $\left(x S+q q^{\prime}\right) p x^{-1}=q$, from (41), so that $p x^{-1}=\left(x S+q q^{\prime}\right)^{-1} q$. Hence, substituting in (42), we regain symmetry via

$$
A=\left(x S+q q^{\prime}\right)^{-1}-x^{-2} p p^{\prime}
$$


which is easily calculated for any given values of $x$ and $p$ once the demand functions are known. For completeness, note the dual of (43) is

$$
x S=\left(A+x^{-2} p p^{\prime}\right)^{-1}-q q^{\prime} .
$$

Finally, in formulae such as (37), it is necessary to evaluate terms such as $\left(u-u^{*}\right) \partial \log \left(a_{i} / a_{j}\right) / \partial u$. From (6), taking logarithms and subtracting

$$
\log \left(p_{i} / p_{j}\right)=\log a_{i}\{u, \nabla c(u, p)\}-\log a_{j}\{u, \nabla c(u, p)\} .
$$

Consider now an infinitesimal change $d \log p_{0}$ (corresponding to $\left.\left(p_{0}-p_{0}^{*}\right) / p_{0}\right)$ and the corresponding $d u=\left(T-q_{0}\right) \partial u / \partial q_{0} \cdot d \log p_{0}$. Hence, differentiating (45) with respect to $u$,

$$
\begin{aligned}
\frac{\partial \log \left(a_{i} / a_{j}\right)}{\partial u} d u & =\sum_{k} \frac{\partial \log \left(a_{i} / a_{j}\right)}{\partial q_{k}} \cdot \frac{\partial q_{k}}{\partial u} \cdot d u \\
& =\sum_{k} \frac{\partial \log \left(a_{i} / a_{j}\right)}{\partial q_{k}} \cdot \frac{\partial q_{k}}{\partial x} \cdot p_{0} q_{0} d \log p_{0},
\end{aligned}
$$

the last term of which contains only the Antonelli matrix and quantities which can be directly estimated.

The last remaining problem, and by far the most difficult, is the estimation of $S$ itself, particularly those elements measuring the substitution between goods and leisure. Although several authors have recently used time-series data to estimate complete systems of commodity and leisure demand equations (see e.g. Abbott and Ashenfelter [1], Phlips [19], and Darrough [4]), there are several problems in using their results. The first is that the difficulty of estimating the separate effects in collinear time-series of wages, prices, and transfer income, makes it necessary to use restrictive and largely untested assumptions. For example, the study by Phlips uses forms of the linear expenditure system which, by incorporating additive separability and linear Engel curves, assume values for the very quantities we wish to measure and guarantee by assumption a uniform tax structure. More general models avoid this difficulty but are conditional on the assumption that individuals are indeed free to vary their supply of labor given an exogenously determined wage. For many, if not most workers, such an assumption is implausible. Clearly, many workers can alter their working conditions by negotiation, either individually or through unions, and wages and prices will play a part in this process. But this is far from a justification for measuring the substitution effects of goods prices on labor supply by regressing commodity and leisure demands on prices and wages. A more hopeful approach would seem to lie in making the opposite assumption, that in any given period, hours worked are outside the worker's control. In such a framework, leisure is preset at some "ration" level and commodity demands are preconditioned on this value. Provided the pre-set level varies over time, or in cross-sections from household to household, rationing theory tells us that we should be able to identify the substitution effects by comparing the effects on commodity demands of changes 
in the ration level with utility held constant. Some of the details of how this should be done, as well as how to treat a sample in which there are observations of both rationing and of free choice, are discussed in Deaton [7] and Deaton and Muellbauer [9].

\section{CONCLUSIONS}

In this paper, rules for optimal differential commodity taxes have been derived for the three different cases usually studied in the literature: the one consumer economy, the unidimensional continuum of consumers economy, and the finite number of discrete consumers economy. In each case, duality theory has been systematically used to give formulae relating to the tax rates themselves rather than to the effects of the taxes on quantities consumed. While these rules do not give explicit forms for the tax rates since these implicitly enter into the other variables, they are nevertheless capable of yielding fresh insights about the structure of an optimal tax general equilibrium. In particular, in all three models, differences in commodity tax rates are seen to be linked to differences in the degree of luxury and differences in the degree of complementarity with leisure. The effects of assuming either weak or quasi-separability are studied for each of the three cases and it is shown that although separability between goods and leisure in all cases simplifies the tax rule, in some cases reducing it to a prescription for uniform taxes, the structure of taxes, whether uniform, regressive or progressive, depends crucially on exactly how separability is formulated. It is thus of central importance that empirical work directed towards providing parameters for evaluating optimal tax formulae should employ functional forms sufficiently general to allow measurement rather than assumption to determine the structure of taxes.

\section{University of Bristol}

Manuscript received September, 1979; revision received May, 1980.

\section{REFERENCES}

[1] Abbott, M., and O. Ashenfelter: "Labour Supply, Commodity Demand, and the Allocation of Time," Review of Economic Studies, 43(1976), 389-411.

$\rightarrow$ Atkinson, A. B., AND J. E. Stiglitz: "The Structure of Indirect Taxation and Economic Efficiency," Journal of Public Economics, 1(1972), 97-119.

[3] - $\rightarrow-$ : "The Design of Tax Structure: Direct Versus Indirect Taxation," Journal of Public Economics, 6(1976), 55-75.

[4] Darrough, M. N.: "A Model of Consumption and Leisure in an Intertemporal Framework: A Systematic Treatment Using Japanese Data," International Economic Review, 18(1977), 677696.

[5] Deaton, A. S.: "The Distance Function and Consumer Behaviour with Applications to Index Numbers and Optimal Taxation," Review of Economic Studies, 46(1979), 391-405.

[6] - : "Optimally Uniform Commodity Taxes," Economics Letters, 2(1976), 357-361.

[7] —_: "Theoretical and Empirical Approaches to Consumer Demand Under Rationing," in Essays in The Theory and Measurement of Consumer Behavior, ed. by A. S. Deaton, Cambridge: Cambridge University Press, 1981. 
[8] Deaton, A. S., And J. Muellbauer: Economics and Consumer Behavior. New York: Cambridge University Press, 1980.

[9] — : "Functional Forms for Labor Supply and Commodity Demands with and without Quantity Restrictions," Econometrica, forthcoming.

[10] Diamond, P. A.: “A Many-Person Ramsey Tax Rule,” Journal of Public Economics, 5(1975), $335-342$.

[11] Diewert, W. E.: "On Symmetry Conditions for Market Demand Functions: A Review and Some Extensions," Review of Economic Studies, 47(1980), 595-601.

[12] Gorman, W. M.: "Quasi-separable Preferences, Costs, and Technologies," University of North Carolina, Chapel Hill, mimeo, 1970.

[13] —_: "Tricks with Utility Functions," in Essays in Economic Analysis, ed. by M. Artis and R. Nobay. Cambridge: Cambridge University Press, 1976.

[14] Guesnerie, R.: "The Distribution of Free Commodities as a Policy Tool," presented to Econometric Society European Meetings, Geneva, September, 1978.

[15] Hicks, J. R.: A Revision of Demand Theory. Oxford: Oxford University Press, 1956.

[16] McFadden, D.: "Cost, Revenue and Profit Functions," in Production Economics: A Dual Approach to Theory and Applications, ed. by M. Fuss and D. McFadden. Amsterdam: North-Holland, 1978.

[17] Mirrlees, J. A.: "The Theory of Optimal Taxation," in Handbook of Mathematical Economics, ed. by K. J. Arrow and M. Intriligator. Amsterdam: North Holland, 1981.

[18] Muellbauer, J.: "Linear Aggregation in Neoclassical Labour Supply," Review of Economic Studies, 48(1981), 21-36.

[19] PhLIPS, L.: "The Demand for Leisure and Money," Econometrica, 46(1978), 1025-1043.

[20] Roberts, K.: "The Treatment of the Poor under Tax/Transfer Schemes," Massachusetts Institute of Technology, mimeo, 1978. 